\title{
Importance of transboundary transport of biomass burning emissions to regional air quality in Southeast Asia during a high fire event
}

B. Aouizerats et al.

Correspondence to: B. Aouizerats (benjamin.aouizerats@vu.nl) 


\section{NOAA HYSPLIT MODEL}

Concentration (mass $/ \mathrm{m} 3$ ) averaged between $0 \mathrm{~m}$ and $100 \mathrm{~m}$ Integrated from 140015 Oct to 020016 Oct 06 (UTC)

PM25 Release started at 140015 Oct 06 (UTC)

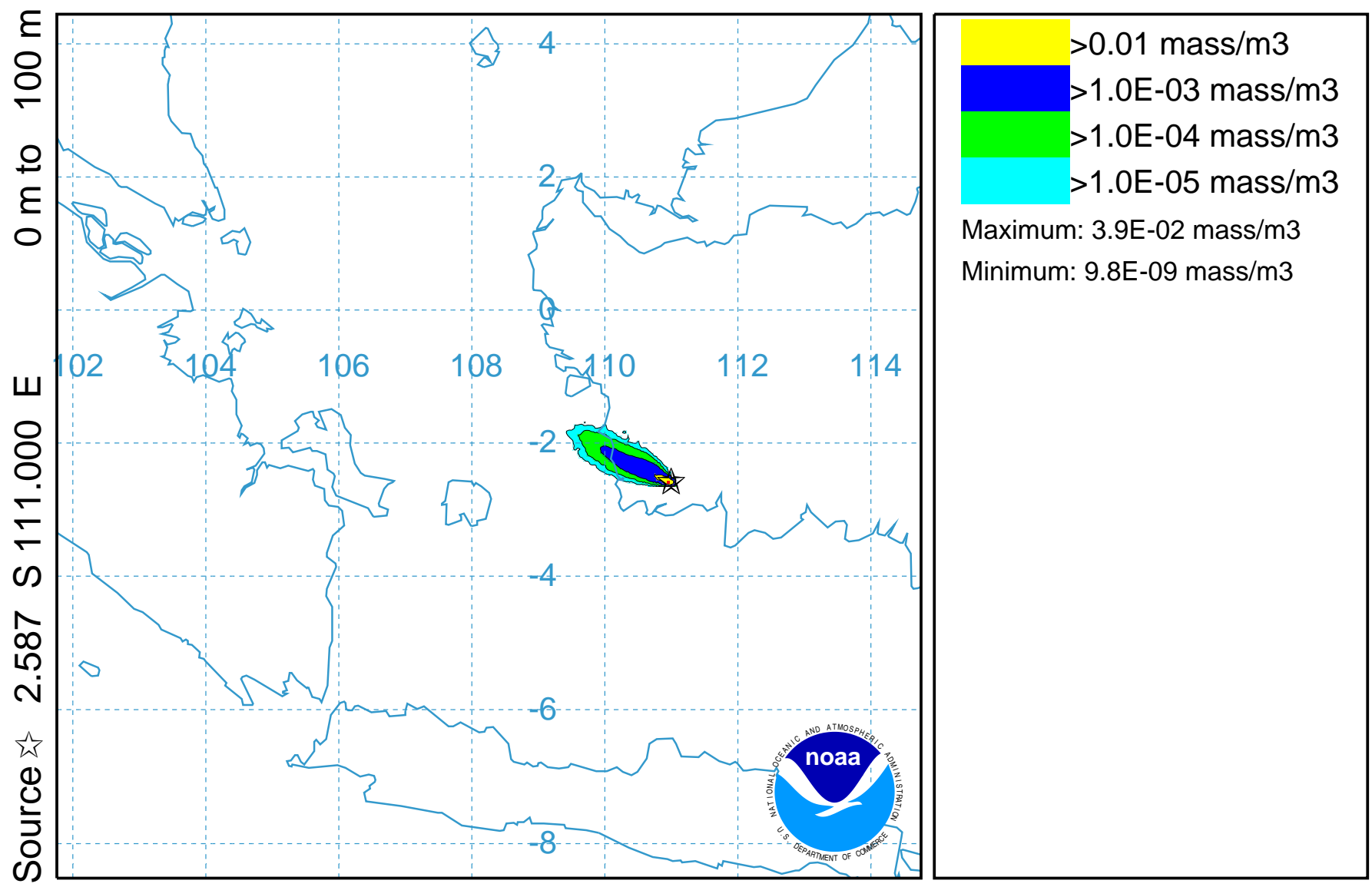

\section{GDAS METEOROLOGICAL DATA}

Job ID: 21377 Job Start: Wed Nov 5 12:22:50 UTC 2014

Release: lat.: -2.587000 lon.: 111.000000 Hgt: 0 to $100 \mathrm{~m}$

Pollutant: PM25 - Unspecified

Release Quantity: 100000000000 mass Start: 061015140 Duration: 84 hrs, 0 m

Pollutant Averaging/lntegration Period: $12 \mathrm{hrs}$ and $0 \mathrm{~min}$

Dry Deposition rate: $0.1 \mathrm{~cm} / \mathrm{s}$ Wet Removal: None \#Part: 94000

Meteorology: 0000Z 15 Oct 2006 - GDAS1

This is not a NOAA product. It was produced by: unknown 


\section{NOAA HYSPLIT MODEL}

Concentration (mass $/ \mathrm{m} 3$ ) averaged between $0 \mathrm{~m}$ and $100 \mathrm{~m}$ Integrated from 020016 Oct to 140016 Oct 06 (UTC)

PM25 Release started at 140015 Oct 06 (UTC)

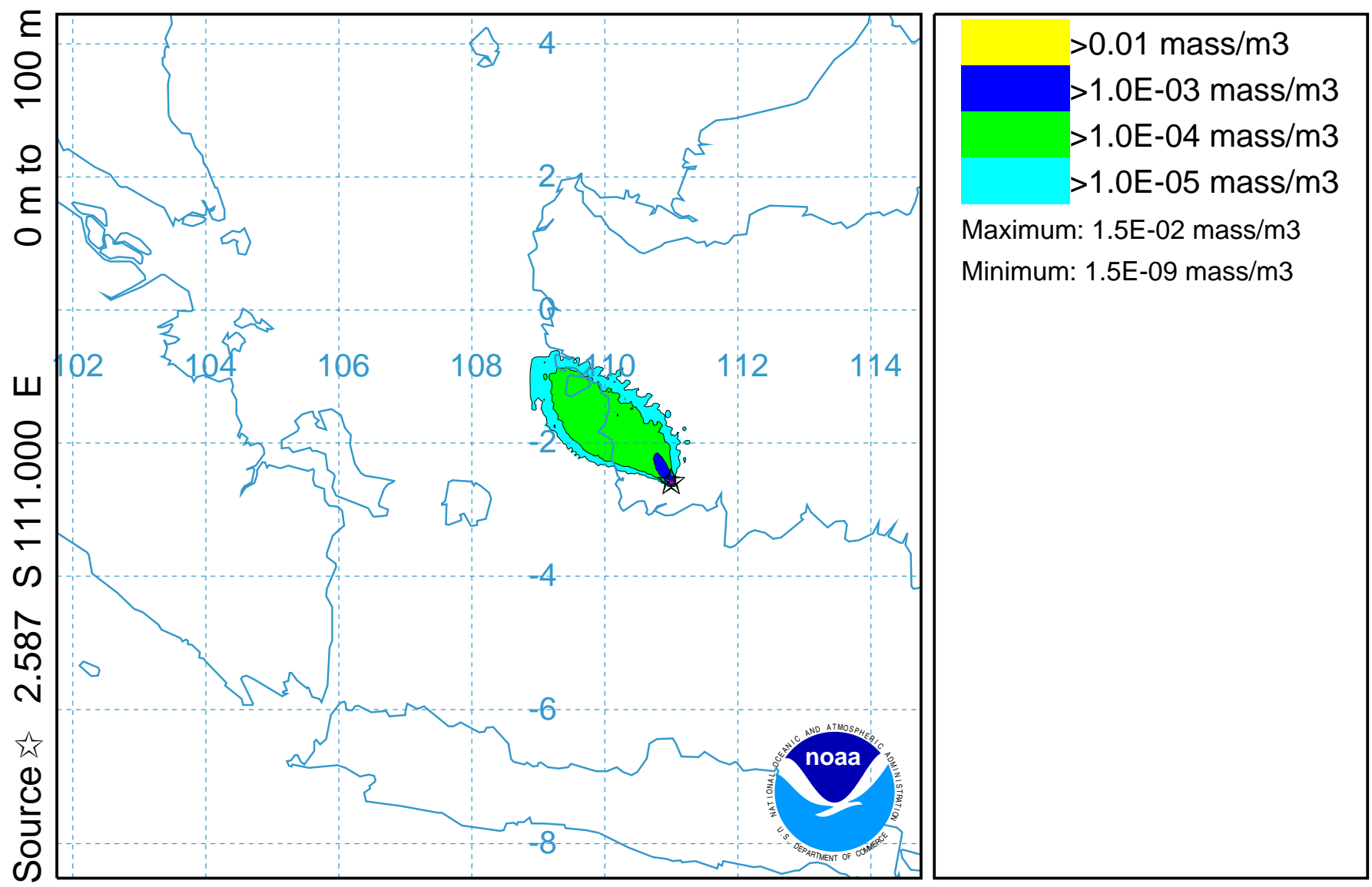

\section{GDAS METEOROLOGICAL DATA}

Job ID: 21377 Job Start: Wed Nov 5 12:22:50 UTC 2014

Release: lat.: -2.587000 lon.: 111.000000 Hgt: 0 to $100 \mathrm{~m}$

Pollutant: PM25 - Unspecified

Release Quantity: 100000000000 mass Start: 061015140 Duration: 84 hrs, 0 m

Pollutant Averaging/lntegration Period: $12 \mathrm{hrs}$ and $0 \mathrm{~min}$

Dry Deposition rate: $0.1 \mathrm{~cm} / \mathrm{s}$ Wet Removal: None \#Part: 94000

Meteorology: 0000Z 15 Oct 2006 - GDAS1

This is not a NOAA product. It was produced by: unknown 


\section{NOAA HYSPLIT MODEL}

Concentration (mass $/ \mathrm{m} 3$ ) averaged between $0 \mathrm{~m}$ and $100 \mathrm{~m}$ Integrated from 140016 Oct to 020017 Oct 06 (UTC)

PM25 Release started at 140015 Oct 06 (UTC)

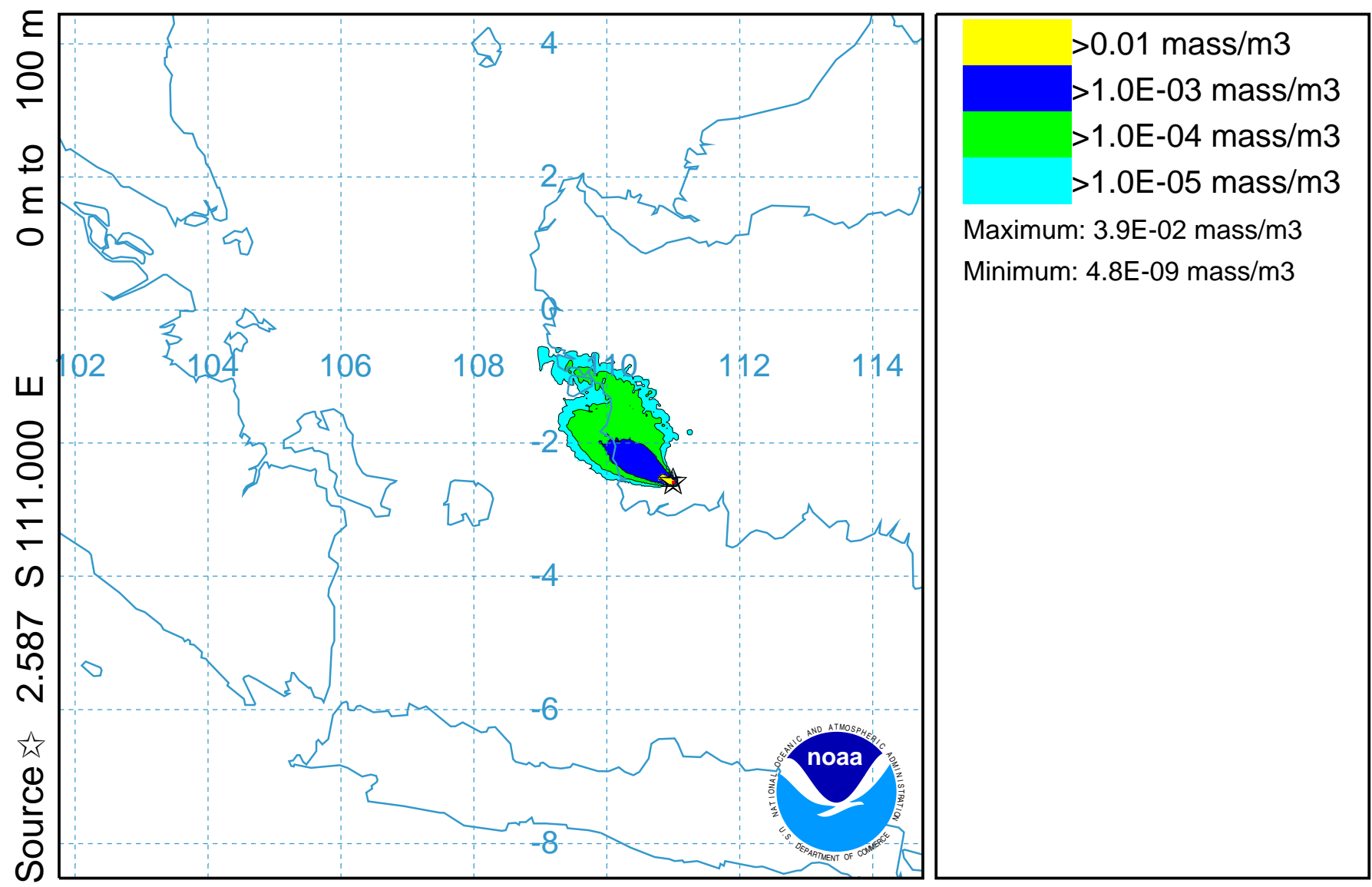

\section{GDAS METEOROLOGICAL DATA}

Job ID: 21377 Job Start: Wed Nov 5 12:22:50 UTC 2014

Release: lat.: -2.587000 lon.: 111.000000 Hgt: 0 to $100 \mathrm{~m}$

Pollutant: PM25 - Unspecified

Release Quantity: 100000000000 mass Start: 061015140 Duration: 84 hrs, 0 m

Pollutant Averaging/lntegration Period: $12 \mathrm{hrs}$ and $0 \mathrm{~min}$

Dry Deposition rate: $0.1 \mathrm{~cm} / \mathrm{s}$ Wet Removal: None \#Part: 94000

Meteorology: 0000Z 15 Oct 2006 - GDAS1

This is not a NOAA product. It was produced by: unknown 


\section{NOAA HYSPLIT MODEL}

Concentration (mass $/ \mathrm{m} 3$ ) averaged between $0 \mathrm{~m}$ and $100 \mathrm{~m}$ Integrated from 020017 Oct to 140017 Oct 06 (UTC)

PM25 Release started at 140015 Oct 06 (UTC)

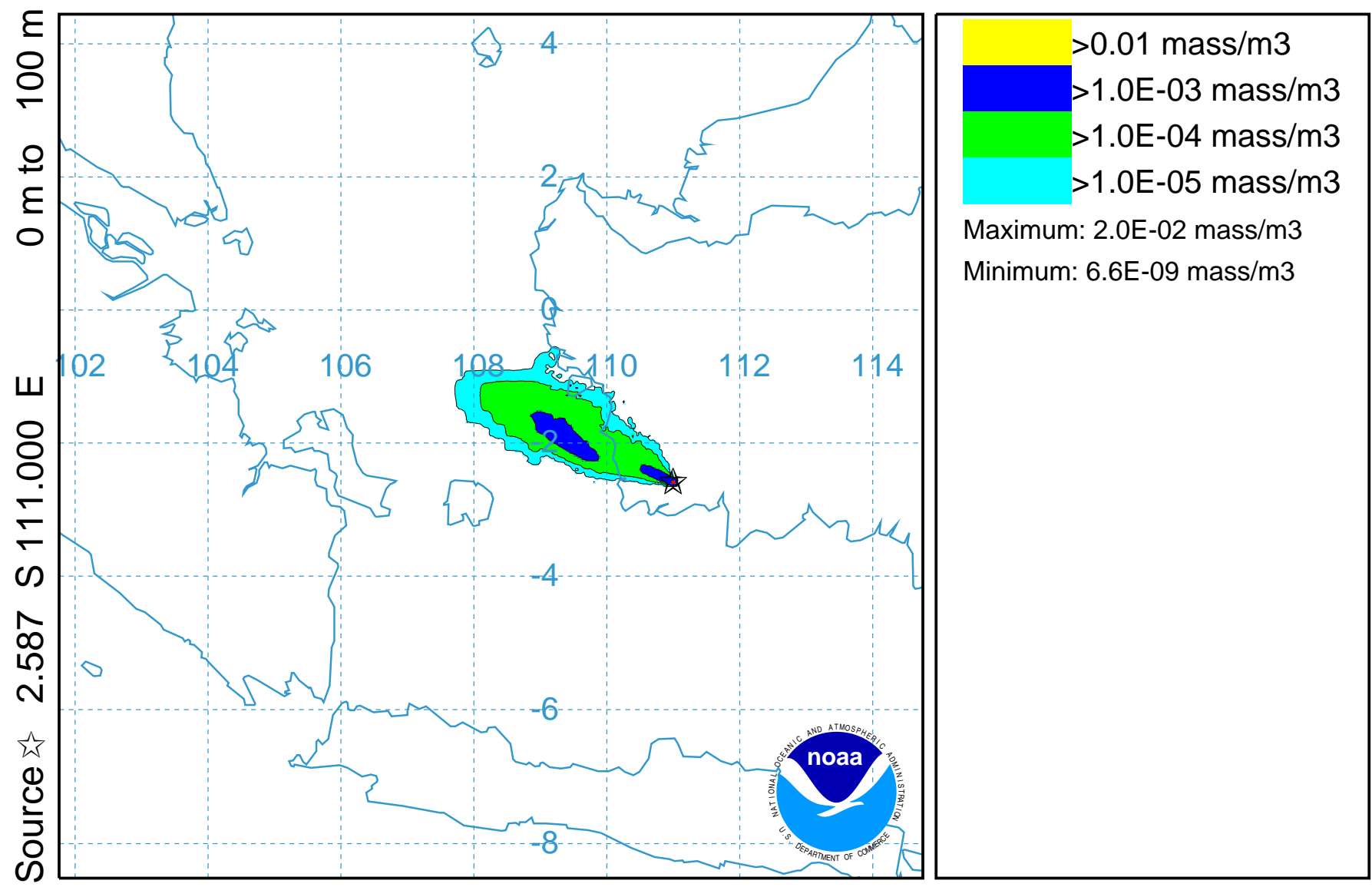

\section{GDAS METEOROLOGICAL DATA}

Job ID: 21377 Job Start: Wed Nov 5 12:22:50 UTC 2014

Release: lat.: -2.587000 lon.: 111.000000 Hgt: 0 to $100 \mathrm{~m}$

Pollutant: PM25 - Unspecified

Release Quantity: 100000000000 mass Start: 061015140 Duration: 84 hrs, 0 m

Pollutant Averaging/lntegration Period: $12 \mathrm{hrs}$ and $0 \mathrm{~min}$

Dry Deposition rate: $0.1 \mathrm{~cm} / \mathrm{s}$ Wet Removal: None \#Part: 94000

Meteorology: 0000Z 15 Oct 2006 - GDAS1

This is not a NOAA product. It was produced by: unknown 


\section{NOAA HYSPLIT MODEL}

Concentration (mass $/ \mathrm{m} 3$ ) averaged between $0 \mathrm{~m}$ and $100 \mathrm{~m}$ Integrated from 140017 Oct to 020018 Oct 06 (UTC)

PM25 Release started at 140015 Oct 06 (UTC)

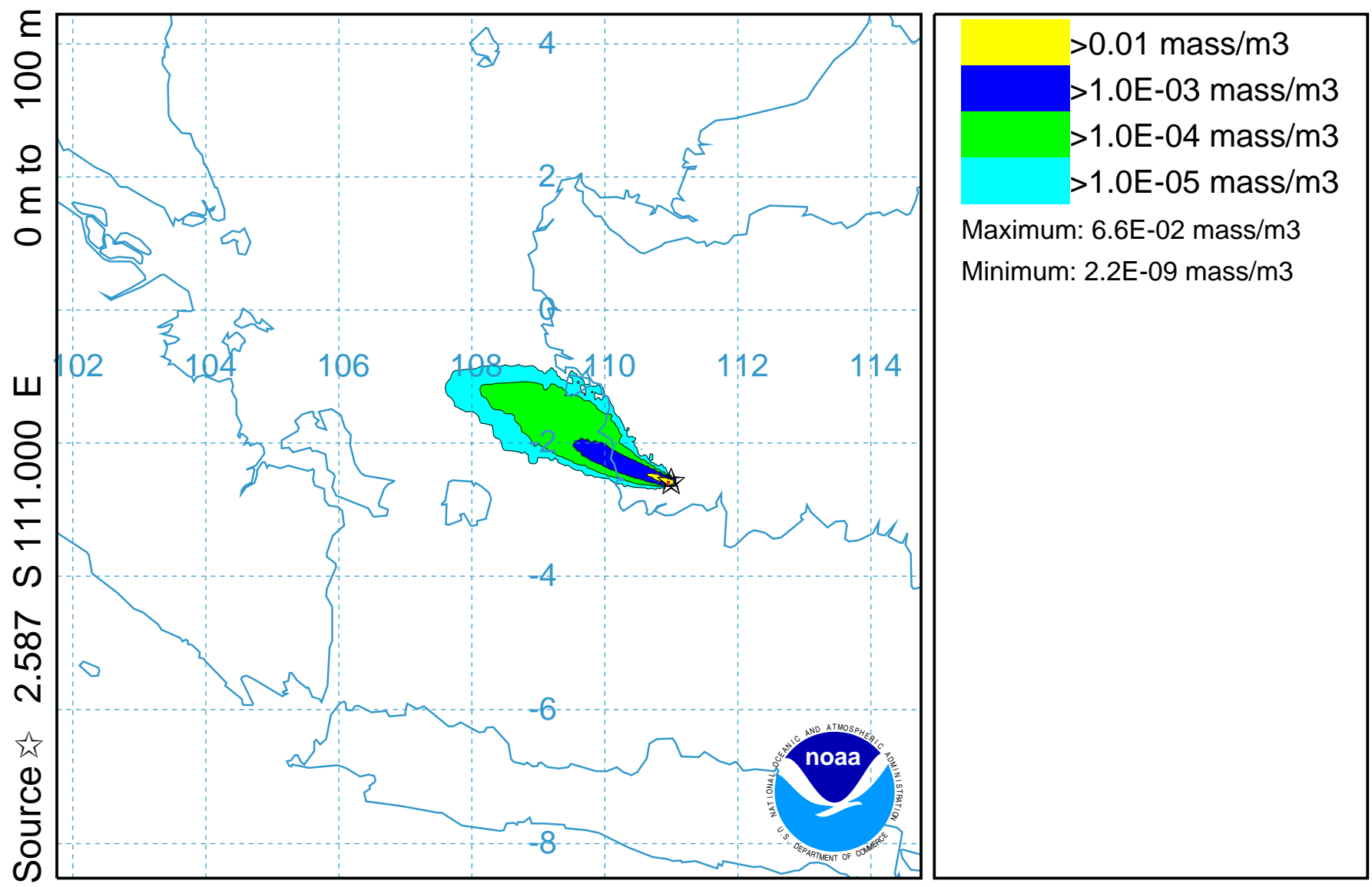

\section{GDAS METEOROLOGICAL DATA}

Job ID: 21377 Job Start: Wed Nov 5 12:22:50 UTC 2014

Release: lat.: -2.587000 lon.: 111.000000 Hgt: 0 to $100 \mathrm{~m}$

Pollutant: PM25 - Unspecified

Release Quantity: 100000000000 mass Start: 061015140 Duration: 84 hrs, 0 m

Pollutant Averaging/lntegration Period: $12 \mathrm{hrs}$ and $0 \mathrm{~min}$

Dry Deposition rate: $0.1 \mathrm{~cm} / \mathrm{s}$ Wet Removal: None \#Part: 94000

Meteorology: 0000Z 15 Oct 2006 - GDAS1

This is not a NOAA product. It was produced by: unknown 


\section{NOAA HYSPLIT MODEL}

Concentration (mass $/ \mathrm{m} 3$ ) averaged between $0 \mathrm{~m}$ and $100 \mathrm{~m}$ Integrated from 020018 Oct to 140018 Oct 06 (UTC)

PM25 Release started at 140015 Oct 06 (UTC)

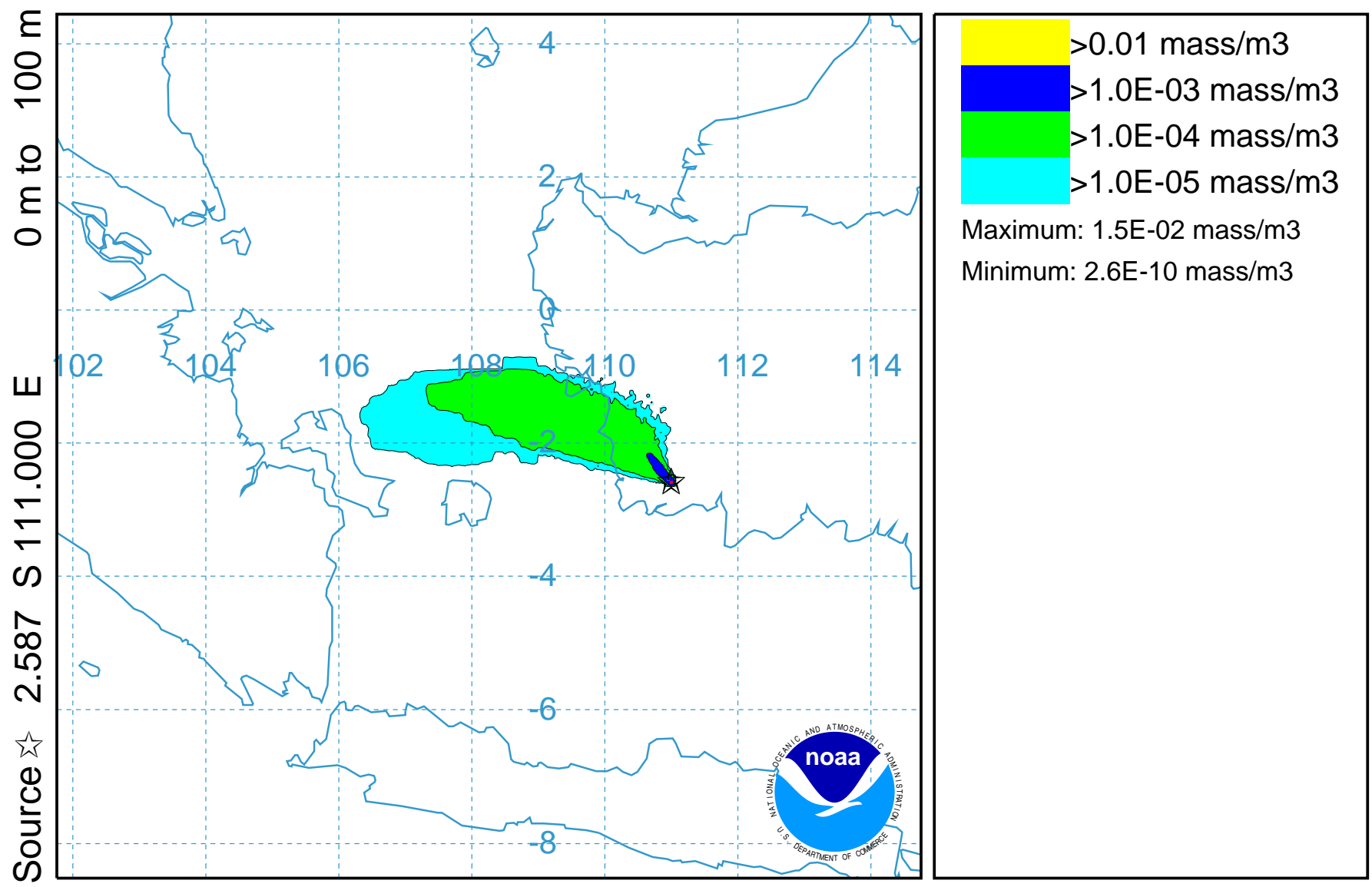

\section{GDAS METEOROLOGICAL DATA}

Job ID: 21377 Job Start: Wed Nov 5 12:22:50 UTC 2014

Release: lat.: -2.587000 lon.: 111.000000 Hgt: 0 to $100 \mathrm{~m}$

Pollutant: PM25 - Unspecified

Release Quantity: 100000000000 mass Start: 061015140 Duration: 84 hrs, 0 m

Pollutant Averaging/lntegration Period: $12 \mathrm{hrs}$ and $0 \mathrm{~min}$

Dry Deposition rate: $0.1 \mathrm{~cm} / \mathrm{s}$ Wet Removal: None \#Part: 94000

Meteorology: 0000Z 15 Oct 2006 - GDAS1

This is not a NOAA product. It was produced by: unknown 


\section{NOAA HYSPLIT MODEL}

Concentration (mass $/ \mathrm{m} 3$ ) averaged between $0 \mathrm{~m}$ and $100 \mathrm{~m}$ Integrated from 140018 Oct to 020019 Oct 06 (UTC)

PM25 Release started at 140015 Oct 06 (UTC)

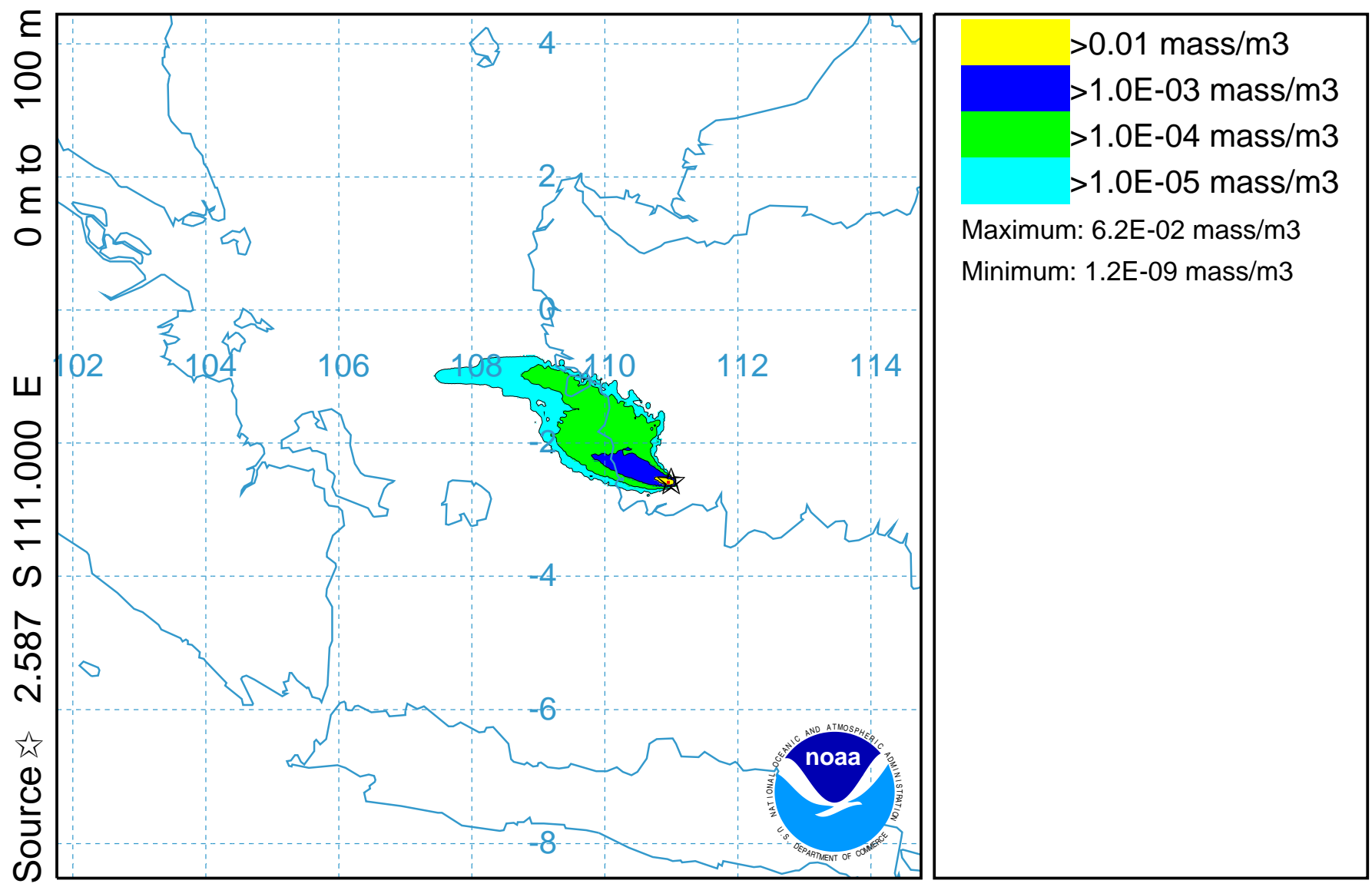

\section{GDAS METEOROLOGICAL DATA}

Job ID: 21377 Job Start: Wed Nov 5 12:22:50 UTC 2014

Release: lat.: -2.587000 lon.: 111.000000 Hgt: 0 to $100 \mathrm{~m}$

Pollutant: PM25 - Unspecified

Release Quantity: 100000000000 mass Start: 061015140 Duration: 84 hrs, 0 m

Pollutant Averaging/Integration Period: $12 \mathrm{hrs}$ and $0 \mathrm{~min}$

Dry Deposition rate: $0.1 \mathrm{~cm} / \mathrm{s}$ Wet Removal: None \#Part: 94000

Meteorology: 0000Z 15 Oct 2006 - GDAS1

This is not a NOAA product. It was produced by: unknown 